\title{
More Accurate Analysis of Sum-Product Decoding of LDPC codes Using a Gaussian Approximation
}

\author{
Francesca Vatta, Member, IEEE, Alessandro Soranzo, and Fulvio Babich, Senior Member, IEEE
}

\begin{abstract}
This letter presents a more accurate mathematical analysis, with respect to the one performed in Chung et al.'s 2001 paper, of belief-propagation decoding for Low-Density ParityCheck (LDPC) codes on memoryless Binary Input - Additive White Gaussian Noise (BI-AWGN) channels, when considering a Gaussian Approximation (GA) for message densities under density evolution. The recurrent sequence, defined in Chung et al.'s 2001 paper, describing the message passing between variable and check nodes, follows from the GA approach and involves the function $\phi(x)$, therein defined, and its inverse. The analysis of this function is here resumed and studied in depth, to obtain tighter upper and lower bounds on it. Moreover, unlike the upper bound given in the above cited paper, the tighter upper bound on $\phi(x)$ is invertible. This allows a more accurate evaluation of the asymptotical performance of sum-product decoding of LDPC codes when a GA is assumed.
\end{abstract}

Index Terms - LDPC codes, threshold, Gaussian approximation, density evolution, sum-product algorithm, upper bound.

\section{INTRODUCTION}

Low Density Parity Check (LDPC) codes are a class of channel block codes first introduced in 1960 by Gallager [1]. Due to the technical limits of that age, they were scarcely considered for almost 30 years, and were re-invented in the mid 1990s by, among the others, Luby et al. [2]. Since their performance can approach the Shannon limit, they were quickly included in modern standards such as IEEE802.11, 802.16, 10G-BaseT Ethernet, and Digital Video Broadcasting.

LDPC codes are still under intense study using tools that require the understanding of the convergence behavior of their iterative decoding process. For example, to design irregular LDPC codes a density-evolution algorithm was used in [3], which, unfortunately, may be unattractive because it requires intensive computations. Another approach is to use extrinsic information transfer (EXIT) charts, which are simpler, because they provide a one-dimensional (1-D) analysis, but ignore information about the probability distribution function (pdf) of messages, resulting in a loss in accuracy.

There have been a number of approaches to 1-D analysis of LDPC codes (see, e.g., [4]), all of them based on the observation that the pdf of the decoders log-likelihood ratio (LLR) messages is approximately Gaussian. This approximation is quite accurate for messages sent from variable nodes, but becomes less accurate for messages sent from check nodes, unless the check nodes degree distribution polynomial $\rho(x)$ (see next section) is concentrated on a few degrees. Despite its limits, the use of the Gaussian Approximation (GA) is

F. Babich and F. Vatta are with DIA, University of Trieste, 34127 Trieste, Italy (e-mail: babich@units.it; vatta@units.it).

A. Soranzo is with DMG, University of Trieste, 34127 Trieste, Italy (e-mail: soranzo@units.it). very useful when an efficient and low complexity method is needed. See its recent use, e.g., to obtain bounds on LDPC decoding thresholds in [5], rate-compatible puncturing patterns for LDPC $\operatorname{codes}^{1}$ in [6] and [7], analytical Bit-error rate (BER) expressions in [8], and UEP LDPC codes in [9].

In [4], following the GA approach, the iterative message passing between variable and check nodes was described mathematically by means of a recurrent sequence involving a function, called $\phi(x)$, therein defined, and its inverse. Its analysis is here resumed and studied in depth, to obtain tighter upper and lower bounds on it. To show their usefulness, a piecewise approximation of $\phi(x)$ is defined, similar but better than the one in [4]. With this new approximation, better GA thresholds can be obtained, closer to the thresholds evaluated with density evolution than the GA thresholds reported in [4].

Moreover, unlike the upper bound in [4], the tighter upper bound on $\phi(x)$ obtained is invertible by means of the Lambert $W$-function. Its invertibility allows, first of all, the extension of the stability condition under the GA to variable node degree distributions $\lambda(x)$ of minimum degree $i_{1}>2$ (see next section): being, in this case, $\lambda^{\prime}(0)=\lambda_{2}=0$, this is useful to design LDPC codes presenting a linear minimum distance growth with the block length with probability 1 [12]. These codes unfortunately cannot reach capacity under iterative decoding, since the achievement of capacity requires $\lambda_{2} \neq 0$. However, in this latter case, the block error probability might converge to a constant [12]. Secondly, the invertibility of the tighter upper bound on $\phi(x)$ allows the computability of the approximated residual word error probability $P_{l}$ at the $l$-th iteration, given in [4], and not only of its rate of convergence.

The letter is organized as follows. Section II reviews the GA for irregular LDPC codes and the function $\phi(x)$ defined in [4], and Section III the bounds on it proven in [4]. In Sections IIIA, III-B, and III-C a tighter upper and lower bound on $\phi(x)$ are given, and a new approximation of $\phi(x)$ is defined, similar but better than the one in [4]. A more general, with respect to the one in [4], stability condition under the GA is presented in Section IV and in Section $\mathrm{V}$ it is shown that the tighter upper bound on $\phi(x)$ allows the computability of $P_{l}$ in the same hypotheses. Finally, Section VI summarizes the results.

\section{GA FOR IRREGULAR LDPC CODES}

In this section, we focus on irregular LDPC codes [3] since they are known to perform better than regular ones [2]. Moreover, it was shown in [4] that message densities can be approximated as Gaussians for regular LDPC codes or

\footnotetext{
${ }^{1}$ Useful in Automatic Repeat-reQuest / Forward Error Correction (ARQ / FEC) schemes [10] and for Unequal Error Protection (UEP) applications [11].
} 
Gaussian mixtures for irregular LDPC codes, and thus, from this viewpoint, regular LDPC codes may be seen as a particular case of irregular ones.

Irregular LDPC codes are defined by specifying the distribution of the node degrees in their Tanner graphs. In particular, in the edge-perspective degree distribution, $\lambda_{i}$ is the fraction of edges in the Tanner graph connecting to a degree$i$ variable node, and $\rho_{j}$ is the fraction of edges connecting to a degree- $j$ check node. To specify the degree distribution, the following polynomials are defined: $\lambda(x):=\sum_{i=i_{1}}^{d_{l}} \lambda_{i} x^{i-1}$ and $\rho(x):=\sum_{j=2}^{d_{r}} \rho_{j} x^{j-1}$, being $d_{l}$ (respectively $d_{r}$ ) the maximum variable (respectively check) node degree. The $d_{l^{-}}$tuple $\left\{\lambda_{i}\right\}$ and $d_{r}$-tuple $\left\{\rho_{j}\right\}$ both add up to 1 .

Consider an irregular LDPC code with edge-perspective degree distributions $\lambda(x)$ and $\rho(x)$. Denote with $v$ the output message of a variable node and with $u$ the output message of a check node. Assuming that irregular LDPC codes message distributions for AWGN channels are approximately Gaussian, since their variance $\sigma^{2}$ is shown in [4] to be related to the mean $m$ by the relation $\sigma^{2}=2 m$, due to the symmetry condition, we can keep the means only. Denoting the means of $u$ and $v$ by $m_{u}^{(l)}$ and $m_{v}^{(l)}$ at the $l$-th iteration, respectively, the LLR message $u_{0}$ from the channel can be assumed to be Gaussian with mean $m_{u_{0}}=2 / \sigma_{n}^{2}$, where $\sigma_{n}^{2}=N_{0} / 2$ is the variance of the channel noise.

For a degree- $i$ variable node at the $l^{t h}$ iteration, the mean of the output yields $m_{v, i}^{(l)}=m_{u_{0}}+(i-1) m_{u}^{(l-1)}$, where $m_{u_{0}}$ is the mean of $u_{0}$ and $m_{u}^{(l-1)}$ is the mean of $u$ at the $(l-1)$-th iteration. Defining $\phi(x)$ as in [4]

$$
\phi(x)= \begin{cases}1-\frac{1}{\sqrt{4 \pi x}} \int_{\mathbb{R}} \tanh \frac{u}{2} \mathrm{e}^{-\frac{(u-x)^{2}}{4 x}} \mathrm{~d} u & \text { if } x>0 \\ 1 & \text { if } x=0\end{cases}
$$

and letting the codeword length tend to infinity (so that we can invoke a cycle-free Tanner graph argument) the check node update rule for an irregular code becomes $m_{u, j}^{(l)}=$ $\phi^{-1}\left(1-\left[1-\sum_{i=i_{1}}^{d_{l}} \lambda_{i} \phi\left(m_{v, i}^{(l)}\right)\right]^{j-1}\right)$. By linearly combining these means for degree- $2, \ldots, d_{r}$ check nodes we get:

$m_{u}^{(l)}=\sum_{j=2}^{d_{r}} \rho_{j} \phi^{-1}\left(1-\left[1-\sum_{i=i_{1}}^{d_{l}} \lambda_{i} \phi\left(m_{u_{0}}+(i-1) m_{u}^{(l-1)}\right)\right]^{j-1}\right)$

Defining $s=m_{u_{0}}$ and $t_{l}=m_{u}^{(l)}$, (2) may be rewritten as

$$
t_{l}=f\left(s, t_{l-1}\right)
$$

where $f(s, t)$ is defined, through the $f_{j}(s, t)$, as:

$$
\begin{gathered}
f_{j}(s, t):=\phi^{-1}\left(1-\left[1-\sum_{i=i_{1}}^{d_{l}} \lambda_{i} \phi(s+(i-1) t)\right]^{j-1}\right) \\
f(s, t):=\sum_{j=2}^{d_{r}} \rho_{j} f_{j}(s, t)
\end{gathered}
$$

\section{THE FUNCTION $\phi(x)$}

For the function (1), these lower and upper bounds have been proved in [4], holding for $x>3$ and for $x>0$, respectively:

$$
\left(1-\frac{3}{x}\right) \sqrt{\frac{\pi}{x}} \mathrm{e}^{-x / 4}<\phi(x)<\left(1+\frac{1}{7 x}\right) \sqrt{\frac{\pi}{x}} \mathrm{e}^{-x / 4}
$$

\section{A. A tighter upper bound on $\phi(x)$}

The following theorem gives a tighter upper bound on $\phi(x)$. Theorem 1

$$
\phi(x)<\sqrt{\frac{\pi}{x}} \mathrm{e}^{-x / 4}=: \psi(x) \quad \forall x>0
$$

Proof: In [4] we find

$$
\phi(x)=4 \sum_{k=0}^{\infty}(-1)^{k} \mathrm{e}^{x\left(k^{2}+k\right)} Q\left(\sqrt{\frac{x}{2}}(1+2 k)\right)
$$

and, using the classical upper bound on the $Q$-function [13] $Q(x)<\frac{1}{x} \frac{1}{\sqrt{2 \pi}} \mathrm{e}^{-x^{2} / 2}, \phi(x)$ can be upper bounded by:

$$
\begin{aligned}
& \phi(x)<4 \sum_{k=0}^{\infty}(-1)^{k} \mathrm{e}^{x\left(k^{2}+k\right)} \sqrt{\frac{2}{x}} \frac{1}{1+2 k} \frac{1}{\sqrt{2 \pi}} \mathrm{e}^{-\frac{1}{2} \frac{x}{2}(1+2 k)^{2}} \\
& =4 \sum_{k=0}^{\infty}(-1)^{k} \frac{1}{1+2 k} \mathrm{e}^{x\left(k^{2}+k-\frac{1}{4}-k-k^{2}\right)} \frac{1}{\sqrt{x}} \frac{1}{\sqrt{\pi}} \\
& =4 \frac{1}{\sqrt{x}} \frac{1}{\sqrt{\pi}} \mathrm{e}^{-x / 4} \sum_{k=0}^{\infty}(-1)^{k} \frac{1}{1+2 k}=4 \frac{1}{\sqrt{x}} \frac{1}{\sqrt{\pi}} \mathrm{e}^{-x / 4} \frac{\pi}{4}
\end{aligned}
$$

where the classical series $\sum_{k=1}^{\infty} \frac{(-1)^{k+1}}{2 k-1}=\frac{\pi}{4}$ has been used ([14], p. 9, $0.232 \# 2$ ), and, in conclusion, the thesis follows.

Notice that (7) is a tighter upper bound than the one in (6), since the term $1 /(7 x)$ of (6) has been replaced in (7) with 0 .

The bound $\psi(x)$ in (7) is invertible by means of the principal branch of the real Lambert $W$-function (which is the inverse of $x \mathrm{e}^{x}$ for $\left.x>-1\right)$. The corresponding upper bound on $\phi^{-1}(y)$ is:

$$
\phi^{-1}(y)<2 W\left(\frac{\pi}{2 y^{2}}\right) \quad 0<y \leq 1
$$

\section{B. A tighter lower bound on $\phi(x)$}

The following theorem gives a tighter lower bound on $\phi(x)$. Theorem 2

$$
\left(1-\frac{\pi^{2}}{4} \frac{1}{x}\right) \sqrt{\frac{\pi}{x}} \mathrm{e}^{-x / 4}<\phi(x) \quad \forall x>\frac{\pi^{2}}{4}
$$

Proof: Using (8) we have, by a classical lower bound on the $Q$-function [13] $\left(\frac{1}{x}-\frac{1}{x^{3}}\right) \frac{1}{\sqrt{2 \pi}} \mathrm{e}^{-x^{2} / 2}<Q(x)$,

$$
\begin{gathered}
\phi(x)>4 \sum_{k=0}^{\infty}(-1)^{k} \mathrm{e}^{x\left(k^{2}+k\right)}\left(\frac{1}{\sqrt{\frac{x}{2}}(1+2 k)}\right. \\
\left.-\frac{1}{\left(\sqrt{\frac{x}{2}}(1+2 k)\right)^{3}}\right) \frac{1}{\sqrt{2 \pi}} \mathrm{e}^{-\frac{1}{2} \frac{x}{2}(1+2 k)^{2}} \\
=4 \mathrm{e}^{-x / 4} \sqrt{\frac{2}{x}} \frac{1}{\sqrt{2 \pi}} \\
\cdot\left(\sum_{k=0}^{\infty}(-1)^{k} \frac{1}{2 k+1}-\frac{2}{x} \sum_{k=0}^{\infty}(-1)^{k} \frac{1}{(2 k+1)^{3}}\right) \\
=4 \mathrm{e}^{-x / 4} \frac{1}{\sqrt{x}} \frac{1}{\sqrt{\pi}}\left(\frac{\pi}{4}-\frac{\pi^{3}}{32} \frac{2}{x}\right)
\end{gathered}
$$

where the series $\sum_{k=1}^{\infty} \frac{(-1)^{k+1}}{2 k-1}=\frac{\pi}{4}$ and $\sum_{k=1}^{\infty} \frac{(-1)^{k+1}}{(2 k-1)^{3}}=$ $\frac{\pi^{3}}{32}$ have been used ([14], p. 9, $0.232 \# 2$ and $0.234 \# 4$, respectively), and, in conclusion, the thesis follows.

Notice that (10) is a tighter lower bound than the one in (6) and has a wider domain. Essentially, the constant 3 of (6) has been replaced in (10) with $\pi^{2} / 4 \approx 2.467$.

In Fig. 1 is reported a graph of $\phi(x)$, obtained applying the Mathematica $^{(R)}$ statement, numerically approximating $\phi(x)$, published in [6] using the analysis of [7], and its old and new lower and upper bounds. 


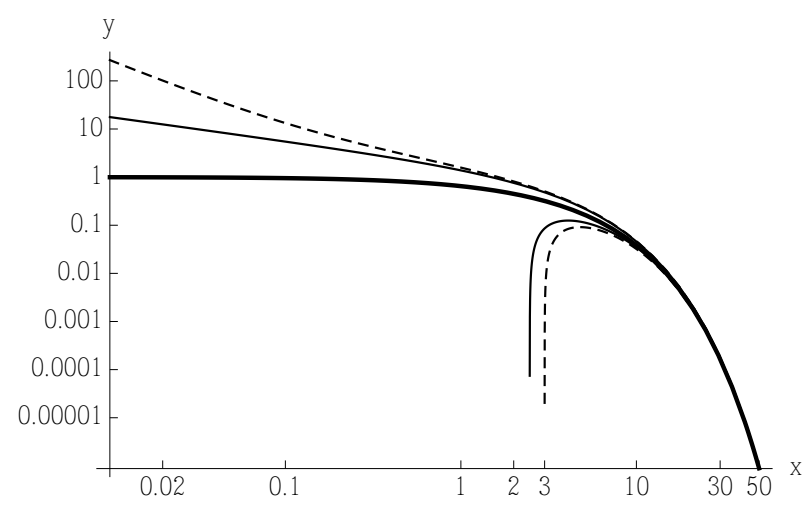

Fig. 1. Graphs of $\phi(x)$ (thick) and its old (dashed) and new (solid) bounds.

\section{A new piecewise approximation of $\phi(x)$}

The function $\phi(x)$ can be approximated with a piecewise defined approximation as the one in [4]. This approximation, defined through an exponential function and the average of the tighter bounds (7) and (10), can be used, instead of the exact values of $\phi(x)$, to speed up the calculations with less sacrifice in accuracy with respect to [4]:

$$
\phi(x) \approx\left\{\begin{array}{lll}
\mathrm{e}^{-0.4527 x^{0.864}+0.0218} & \text { if } & 0 \leq x \leq 10 \\
\sqrt{\frac{\pi}{x}} \mathrm{e}^{-x / 4}\left(1-\frac{\pi^{2}}{8 x}\right) & \text { if } & x>10
\end{array}\right.
$$

With regard to regular LDPC codes, Table I reports in the first 4 columns the values of Table I in [4] (apart from the codes rates, given by $r=1-j / k$ ), in the 5-th the errors of Table I in [4] recalculated with greater precision, in the 6-th the corresponding values $\sigma_{\text {GAnew }}$ computed using (11), and in the 7-th the new errors $\epsilon_{\text {new }}$ in $\mathrm{dB}$ between the new approximated and exact thresholds. As may be seen from the table, all the new computed thresholds are closer to the thresholds evaluated with density evolution than the GA thresholds reported in [4]. Moreover, applying the set of software instructions produced in [15] to evaluate these thresholds, instead of implementing the algorithm sketched in [4], a computational time at least one order of magnitude lower is needed (see [15]).

With regard to irregular LDPC codes, as example we consider the rate-1/2 irregular LDPC code given in Eq. (17) of [4] with degree distributions:

$$
\begin{aligned}
\lambda(x)= & 0.23403 x+0.21242 x^{2}+0.14690 x^{5}+0.10284 x^{6} \\
& +0.30381 x^{19} \\
\rho(x)= & 0.71875 x^{7}+0.28125 x^{8}
\end{aligned}
$$

Applying (11) we get a GA threshold $\sigma_{\mathrm{GAnew}}=0.9538$, closer to the density evolution one $\sigma_{\text {exact }}=0.9669$ (reported in [4]) than the GA threshold $\sigma_{\mathrm{GA}}=0.9473$ computed in [4].

\section{EXTENSION OF THE STABILITY CONDITION}

To extend the stability condition given in [4] to degree distributions $\lambda(x)$ of minimum degree $i_{1} \geq 2$, we can write the following

Theorem 3

As $t \gg$ (as $t$ gets very large, or, equivalently, the probability
TABLE I

APPROXIMATE THRESHOLD VALUES FOR VARIOUS $(j, k)$-REGULAR LDPC CODES.

\begin{tabular}{|c|c|c|c|c|c|c|}
\hline$j$ & $k$ & $\sigma_{\mathrm{GA}}$ & $\sigma_{\text {exact }}$ & $\epsilon[\mathrm{dB}]$ & $\sigma_{\text {GAnew }}$ & $\epsilon_{\text {new }}[\mathrm{dB}]$ \\
\hline \hline 3 & 6 & 0.8747 & 0.8809 & 0.061 & 0.8802 & 0.007 \\
\hline 4 & 8 & 0.8323 & 0.8376 & 0.054 & 0.8374 & 0.002 \\
\hline 5 & 10 & 0.7910 & 0.7936 & 0.028 & 0.7958 & 0.024 \\
\hline 3 & 5 & 1.0003 & 1.0093 & 0.077 & 1.0063 & 0.026 \\
\hline 4 & 6 & 1.0035 & 1.0109 & 0.063 & 1.0094 & 0.013 \\
\hline 3 & 4 & 1.2517 & 1.2667 & 0.103 & 1.2589 & 0.054 \\
\hline 4 & 10 & 0.7440 & 0.7481 & 0.047 & 0.7486 & 0.006 \\
\hline 3 & 9 & 0.7051 & 0.7082 & 0.038 & 0.7097 & 0.019 \\
\hline 3 & 12 & 0.6297 & 0.6320 & 0.031 & 0.6340 & 0.028 \\
\hline
\end{tabular}

of error gets very small), $f(s, t)$ becomes

$$
f(s, t)=2 \sum_{j=2}^{d_{r}} \rho_{j} W\left(A_{j} z(s, t) \mathrm{e}^{z(s, t)}\right)+O\left(t^{-1}\right)
$$

where $z(s, t):=\frac{s+\left(i_{1}-1\right) t}{2}$ and $A_{j}:=\frac{1}{(j-1)^{2} \lambda_{i_{1}}^{2}}$.

Proof: When $t \gg$ since, as shown in Fig. 1, the function $\phi(x)$ has a rapid decrease in $x$, only the first terms of $\sum_{i=i_{1}}^{d_{l}} \lambda_{i} \phi(s+(i-1) t)$ are important in determining $f_{j}(s, t)$ in Eq. (4). Thus, we can simplify that sum to:

$$
\begin{gathered}
\sum_{i=i_{1}}^{d_{l}} \lambda_{i} \phi(s+(i-1) t) \\
=\lambda_{i_{1}} \phi\left(s+\left(i_{1}-1\right) t\right)+O\left(\lambda_{i_{2}} \phi\left(s+\left(i_{2}-1\right) t\right)\right),
\end{gathered}
$$

being $\lambda_{i_{1}}$ and $\lambda_{i_{2}}$ the first and second nonzero $\lambda_{i}$ 's, respectively, and observing that, as regards the irregular LDPC codes having the check nodes degree distribution polynomial $\rho(x)$ concentrated on a few degrees (for which GA results accurate), $\lambda_{i_{2}}$ is never significantly larger than $\lambda_{i_{1}}$ (see, e.g., Table II in [3]), and that they are both very significant. By the expansion

$$
\begin{gathered}
(1-x)^{n}=1-n x+O\left(x^{2}\right) ; \quad x \rightarrow 0 \\
f_{j}(s, t)=\phi^{-1}\left((j-1) \lambda_{i_{1}} \phi\left(s+\left(i_{1}-1\right) t\right)+O\left(\phi\left(s+\left(i_{1}-1\right) t\right)\right)\right)
\end{gathered}
$$

where we used $\phi\left(s+\left(i_{2}-1\right) t\right) \ll \phi\left(s+\left(i_{1}-1\right) t\right)$. Then

$\phi\left(f_{j}(s, t)\right)=(j-1) \lambda_{i_{1}} \phi\left(s+\left(i_{1}-1\right) t\right)+O\left(\phi\left(s+\left(i_{1}-1\right) t\right)\right)$

Using the bound defined in (7),

$\phi\left(f_{j}(s, t)\right)=\sqrt{\frac{\pi}{s+\left(i_{1}-1\right) t}} \mathrm{e}^{-\frac{s+\left(i_{1}-1\right) t-4 \log \left((j-1) \lambda_{i_{1}}\right)}{4}}+O\left(t^{-1}\right)$

and, applying (9),

$f_{j}(s, t)=2 W\left(\frac{s+\left(i_{1}-1\right) t}{2} \mathrm{e}^{\frac{s+\left(i_{1}-1\right) t-4 \log \left((j-1) \lambda_{i_{1}}\right)}{2}}\right)+O\left(t^{-1}\right)$

that can be rewritten as

$$
f_{j}(s, t)=2 W\left(A_{j} z(s, t) \mathrm{e}^{z(s, t)}\right)+O\left(t^{-1}\right)
$$

and applying (5) we get the result.

Applying the method defined in [15], instead of searching the minimum value of the parameter $s=m_{u_{0}}$ granting the convergence of (2), we can solve a problem of quadratic degeneracy. When the partial second derivative $f_{t t}(s, t)$ is $\neq 0$ the problem of quadratic degeneracy is the system of equations

$$
\left\{\begin{array}{l}
f(s, t)=t \\
f_{t}(s, t)=1
\end{array}\right.
$$


being $f_{t}(s, t)$ the partial derivative of $f(s, t)$ with respect to $t$.

Remembering that $\frac{\mathrm{d} W(x)}{\mathrm{d} x}=\frac{1}{x+\mathrm{e}^{W(x)}}$, and applying (15) to (13) and its partial derivative with respect to $t$, we get

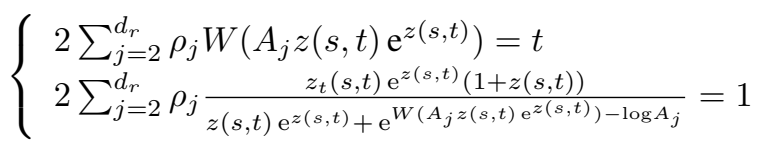

Given the channel and $\rho(x)$, its solution $\left(\lambda_{i_{1}}^{*}, t^{*}\right)$, obtained applying the set of software instructions produced in [15], gives rise to a general stability condition [3] $\lambda_{i_{1}}<\lambda_{i_{1}}^{*}$, valid $\forall i_{1} \geq 2$, unlike the stability condition $\lambda_{2}<\lambda_{2}^{*}$ proved in [4], holding for $i_{1}=2$ only, which is a particular case of this general one. The result of [4] can be derived approximating (14) with $f_{j}(s, t)=2 W\left(\left(z(s, t)+a_{j}\right) e^{z(s, t)+a_{j}}\right)+O\left(t^{-1}\right)$, where $a_{j}:=-2 \log \left((j-1) \lambda_{i_{1}}\right)$. Calling $x:=z(s, t)+a_{j}$, and being $W\left(x e^{x}\right)=x$ for $x>0$, since $f_{j}(s, t)=2 x$, we find the same asymptotical approximation for $f_{j}(s, t)$ of [4]. Applying (5) and getting its partial derivative with respect to $t$, (15) becomes

$$
\left\{\begin{array}{l}
s+\left(i_{1}-1\right) t-4 \log \lambda_{i_{1}}-4 \sum_{j=2}^{d_{r}} \rho_{j} \log (j-1)=t \\
i_{1}-1=1
\end{array}\right.
$$

Its solution is $4 \log \lambda_{2}^{*}=s-4 \sum_{j=2}^{d_{r}} \rho_{j} \log (j-1)$, i.e., we obtain the same $\lambda_{2}^{*}$ given in [4], holding for $i_{1}=2$ only.

Taking as example the rate-1/2 irregular LDPC code (12) and solving (16) with $i_{1}=2$ and $s=2 / \sigma_{\text {GAnew }}^{2}$ derived in Section III-C, we get $\lambda_{2}^{*}=0.2384$. Given the density evolution threshold $\sigma_{\text {exact }}=0.9669$ computed in [4], the maximum stable value of $\lambda_{2}$ is $\lambda_{2}^{*}=\exp \left(1 / 2 \sigma_{\text {exact }}^{2}\right) / \rho^{\prime}(1)=0.2345$ [3]. On the other hand, solving (17) with $s=2 / \sigma_{\mathrm{GA}}^{2}$ (given in [4]) we get $\lambda_{2}^{*}=0.2402$. Thus, solving the more precise system (16) and using the better approximation (11) to evaluate the GA threshold, we get a $\lambda_{2}^{*}=0.2384$ closer to the density evolution one $\lambda_{2}^{*}=0.2345$ than the $\lambda_{2}^{*}=0.2402$ computed solving (17), i.e., using the $\lambda_{2}^{*}$ expression given in [4].

\section{COMPUTABLE APPROXIMATION OF WORD RESIDUAL ERROR PROBABILITY}

The solution of (16) determines, besides the maximum stable value of $\lambda_{i_{1}}, \lambda_{i_{1}}^{*}$, also the convergence abscissa $t^{*}$. The stability condition for the convergence of $t_{l}$ to $\infty$ in (3) with $l \gg$ when $t_{0}$ is large enough [4], is $\lambda_{i_{1}}<\lambda_{i_{1}}^{*} ; t \geq t_{0}=t^{*}$. The asymptotical approximation of $f(s, t)$ given in [4] is $f(s, t)=s+\left(i_{1}-1\right) t-4 \log \lambda_{i_{1}}-4 \sum_{j=2}^{d_{r}} \rho_{j} \log (j-1)+O\left(t^{-1}\right)$ and $\left.4 \log \frac{\lambda_{i_{1}}^{*}}{\lambda_{i_{1}}}\right|_{i_{1}=2} \approx s-4 \log \lambda_{2}-4 \sum_{j=2}^{d_{r}} \rho_{j} \log (j-1)$. Thus, it may be written as $\left.f(s, t)\right|_{i_{1}=2} \approx t+4 \log \frac{\lambda_{2}^{*}}{\lambda_{2}}$, and $t_{l}=\left.f\left(s, t_{l-1}\right)\right|_{i_{1}=2} \approx t_{l-1}+4 \log \frac{\lambda_{2}^{*}}{\lambda_{2}}$. In conclusion, $\left.t_{l}\right|_{i_{1}=2} \approx t_{0}+4 l \log \frac{\lambda_{2}^{*}}{\lambda_{2}}$ with $t_{0}=t^{*}$. This reobtains Eq. (20) in [4] with the constant $c$ (not given in [4]) equal to $t_{0}=t^{*}$.

Applying the approximation of $f(s, t)$ given in [4], and getting $f_{t}(s, t)$, the solution of (17) determines $\lambda_{2}^{*}$ but not $t$ : thus, the constant $c$ of Eq. (20) in [4] cannot be determined. It follows that the asymptotical approximation of the word residual error probability $P_{l}$ at the $l$-th iteration, given in [4], cannot be computed either, because the constants $a, b, d$, and $f$ involved in it depend not only on the degree distributions and $s$, as said in [4], but also on $c$. Instead, the tighter upper bound (7) on $\phi(x)$ allows the calculation of $c=t_{0}$ as solution of (16) for $i_{1} \geq 2$, thus allowing the computability of $P_{l}$ at the $l$-th iteration (and not only the determination of its rate of convergence $\left.\left(\lambda_{2} / \lambda_{2}^{*}\right)^{2 l}\right)$.

\section{Conclusions}

In this letter, the derivation of tighter lower and upper bounds on the function $\phi(x)$ defined in [4] was addressed. To show the usefulness of these two bounds, a new piecewise approximation of $\phi(x)$ has been defined, with which better GA thresholds can be obtained, closer to the thresholds evaluated with density evolution than the GA thresholds given in [4]. Moreover, thanks to the invertible upper bound on $\phi(x)$, it has been possible to extend the stability condition under the GA to degree distributions of minimum degree $i_{1} \geq 2$, useful to design LDPC codes presenting a linear minimum distance growth with the block length.

\section{REFERENCES}

[1] R. G. Gallager, "Low-Density Parity-Check Codes", MIT press Cambridge, 1963.

[2] M. G. Luby, M. Mitzenmacher, M. A. Shokrollahi, and D. A. Spielman, "Improved Low-Density Parity-Check Codes Using Irregular Graphs", IEEE Trans. on Inf. Theory, vol. 47, no. 2, Feb. 2001, pp. 585-598.

[3] T. J. Richardson, A. Shokrollahi, and R. Urbanke, "Design of CapacityApproaching Irregular Low-Density Parity-Check Codes", IEEE Trans. on Inf. Theory, vol. 47, no. 2, Feb. 2001, pp. 619-637.

[4] S.-Y. Chung, T. J. Richardson, and R. Urbanke, "Analysis of SumProduct Decoding of Low-Density Parity-Check Codes Using a Gaussian Approximation", IEEE Trans. on Inf. Theory, vol. 47, no. 2, Feb. 2001, pp. 657-670.

[5] F. Vatta, A. Soranzo and F. Babich, "Low-Complexity Bound on Irregular LDPC Belief-Propagation Decoding Thresholds using a Gaussian approximation", Electronics Letters, Vol. 54, No. 17, Aug. 2018, pp. 1038-1040.

[6] F. Babich, M. Noschese, A. Soranzo, and F. Vatta, "Low Complexity Rate Compatible Puncturing Patterns Design for LDPC Codes", Proc. of the 2017 Int. Conf. SoftCOM, Split, Croatia, Sept. 21-23, 2017.

[7] F. Babich, M. Noschese, and F. Vatta, "Analysis and Design of Rate Compatible LDPC Codes", Proc. of the 27th IEEE Int. Symp. on Personal, Indoor and Mobile Radio Communications - PIMRC '16, Valencia, Spain, September 4-8, 2016, pp. 1-6.

[8] B. S. Tan, K. H. Li, and K. C. Teh, "Bit-error rate analysis of low-density parity-check codes with generalised selection combining over a rayleighfading channel using gaussian approximation", IET Communications, vol. 6, no. 1, 2012, pp. 90-96.

[9] X. Chen and F. C. M. Lau, "Optimization of LDPC Codes with Deterministic UEP Properties", IET Communications, vol. 5, no. 11, 2011, pp. 1560-1565.

[10] F. Babich and F. Vatta,"Turbo codes construction for robust hybrid multitransmission schemes", Journal of Communication Software and Systems (JCOMSS), Vol. 7, No. 4, December 2011, pp. 128-135.

[11] F. Babich, M. D’Orlando, and F. Vatta, "Distortion Estimation Algorithms for Real-Time Video Streaming: an Application Scenario", Proc. of the 2011 Int. Conf. SoftCOM, Split, Croatia, Sept. 15-17, 2011.

[12] C. Di, T. J. Richardson, and R. Urbanke, "Weight Distribution of LowDensity Parity-Check Codes", IEEE Trans. on Inf. Theory, vol. 52, no. 11, Nov. 2006, pp. 4839-4855.

[13] J. M. Wozencraft and I. M. Jacobs, Principles of Communication Engineering. Wiley, New York, 1965.

[14] I. S. Gradshtein and I. M. Ryzhik, Table of Integrals, Series, and Product, Fifth Edition. A. Jeffrey, Editor. Translated from the Russian by Scripta Technica, Inc. - Academic Press, San Diego, 1994.

[15] F. Babich, A. Soranzo, and F. Vatta, "Useful Mathematical Tools for Capacity Approaching Codes Design", IEEE Communications Letters, vol. 21, no. 9, Sept. 2017, pp. 1949-1952. 